\title{
Theoretical Calculation of X-ray Emission from
} Laser-Produced Plasmas*

\author{
D. Duston, R.W. Clark, and J. Davis \\ Naval Research Laboratory \\ Washington, D.C.
}

The interaction of an Intense short-wavelength laser beam with a solid foil target is a topic which has been studied vigorously for many years. Many inherent problems st111 exist, however, in our understanding of observed experimental data. In particular, the problem of transport through the target material of the deposited laser energy continues to be the focus of many investigations. Although most. of the work has been dominated by particle transport phenomena, it was shown in a previous paper that radiation processes and photon transport often play a major role in the redistribution of the deposited energy in laser driven plasmas. In this presentation, we continue our investigation of the role of atomic physics and radiative emission in laser-produced plasmas by studying the effects of these physical processes as a function of varying initial conditions for both aluminum and carbon foil targets.

The main computational tool employed in this study was a $1-D$ radiation/hydrodynamics model in which the fluid equations conserving mass, momentum, and energy are solved self-consistently with the Ionization dynamics and radiation transport to yield accurate information regarding the interaction between photons and plasma particles.

The ionization model is based on collisional-radiative equilibrium (CRE) in which the population densities of ground and excited states are calculated by considering the most important particle and photon collisional processes populating them and solving for a time-independent solution of the set of atomic rate equations. A detailed atomic level structure for aluminum is used which includes all 14 ground states, 84 excited levels, 97 free-bound continuum edges, and 144 averaged emission lines (more than $3000 \mathrm{n} \ell^{-} \mathrm{n}^{-} \ell^{-}$transitions have been included).

The carbon model has not been as thoroughly documented as the aluminum one but includes all 7 ground states, 50 exclted states, 56 free-bound continuum edges, and 107 averaged emission lines. The level structure for carbon is as complete as for aluminum but, of course, it has fewer ions and consequently fewer excited states and lines. The collisional processes treated in the model include electron impact excitation and deexcitation, electron impact ionization and recombination, radiative recombination, dielectronic recombination, and spontaneous radiative decay. The radiation transport algorithm is based on a combination of probabilistic and frequency-by-frequency ray trace techniques and takes account of photolonization, inverse bremsstrahlung (of non-laser photons), photoexcitation and stimulated emission processes. Of extreme 1mportance in stopping the soft $x$-rays in the dense foil regions is inner-shell opacity due to photolontzation of inner-orbit electrons. This is treated along with the valence-electron 
photolonization scheme in a unique way that allows the absorption edges to shift as the dense region heats up and ionizes. The opacity calculation is solved iteratively at each time step with the ionization calculation. Thus, the radiative transfer scheme treats opacity in the plasma self-consistently with the ionization dynamics and hydrodynamics.

In this study, six seperate calculations were performed; in each, one parameter (laser irradiance, laser wavelength, target material, laser pulse width, or target thickness) was varied from the "standard calculation" in which the initial conditions were assumed to be $10^{13}$ watts $/ \mathrm{cm}^{2}, 0.35 \mathrm{um}$ laser light, $3.0 \mathrm{nsec}$ pulse width (fwhm), and an $8.0 \mu$ m-thick aluminum foil target.

Several important conclusions were reached based on the results of these calculations. First, it was verified that the radiation emitted from the plasma is a major energy loss mechanism, accounting for $27-38 \%$ of the deposited laser energy for the aluminum targets over the parameter range studied. In addition, the radiation conversion efficlency for the carbon target calculation was $28 \%$, putting to rest the previous belief held throughout the community that radiation was negligible when studying thin carbon targets.

Second, it was found that the radiation spectrum obtained from the rearside of the foll was strongly dependent on initial conditions. In particular, low-energy ( hv < 100eV) bremsstrahlung photons are emitted directly from the backside of the target, and their spectral distribution and intensity directly reflects the plasma temperature of this region. Thus, as the foll thickness, material, and laser irradiance are changed, the rearside spectrum responds drastically.

Finally, interesting effects on the plasma profiles were found by neglecting the radiation loss term in the energy equation and turning off the photon transport. Comparisions of the calculations with and without radiation showed clear and rather significant differences in the plasma density and temperature profiles. The region containing the laser critical surface is the point of greatest emission; thus, the cooling effect of the radiation here markedly reduces the local temperatures. The resulting effect on the local density is to reduce the gradient substantially in the ablation region, increasing the ablation surface-to-critical surface seperation. of course, this parameter is a very important one in the calculation of thermal conduction, lateral thermal smoothing, and fluid instability growth. This casts doubt on the results of a calculation which employs only approximate fonization-radiation models or, worse, neglects the impact of radiation on the plasma totally.

1 D. Duston, R. W. Clark, J. Davis, and J. P. Apruzese, Phys. Rev. A 27,1441 (1983).

* Supported by the Office of Naval Research. 